\title{
Regulation of miR-1-Mediated
} Connexin 43 Expression and Cell Proliferation in Dental Epithelial Cells

\author{
Tomoaki Nakamura1, Tsutomu Iwamoto², Hannah M. Nakamura ${ }^{3}$, Yuki Shindo ${ }^{4}$, \\ Kan Saito' ${ }^{1}$, Aya Yamada1, Yoshihiko Yamada ${ }^{5}$, Satoshi Fukumoto' ${ }^{1}$ and \\ Takashi Nakamura4*
}

1 Division of Pediatric Dentistry, Department of Oral Health and Development Sciences, Tohoku University Graduate School of Dentistry, Sendai, Japan, ${ }^{2}$ Department of Pediatric Dentistry, Institute of Biomedical Sciences, Tokushima University Graduate School, Tokushima, Japan, ${ }^{3}$ Division of Nephrology and Endocrinology, Tohoku Medical and Pharmaceutical University, Sendai, Japan, ${ }^{4}$ Division of Molecular Pharmacology and Cell Biophysics, Department of Oral Biology, Tohoku University Graduate School of Dentistry, Sendai, Japan, ${ }^{5}$ Laboratory of Cell and Developmental Biology, National Institute of Dental and Craniofacial Research, NIH, Bethesda, MD, United States

\section{OPEN ACCESS}

Edited by:

Dominic C. Voon,

Kanazawa University, Japan

Reviewed by:

Ashish K. Gadicherla,

Federal Institute for Risk Assessment

(BfR), Germany

Michele D'Amico,

University of Campania Luigi Vanvitelli,

Italy

*Correspondence:

Takashi Nakamura

takashi.nakamura.d2@tohoku.ac.jp

Specialty section:

This article was submitted to

Cell Growth and Division,

a section of the journal

Frontiers in Cell and Developmental

Biology

Received: 21 November 2019

Accepted: 26 February 2020

Published: 17 March 2020

Citation:

Nakamura T, Iwamoto T, Nakamura HM, Shindo Y, Saito $K$, Yamada A, Yamada Y, Fukumoto $S$ and Nakamura T (2020) Regulation

of miR-1-Mediated Connexin 43 Expression and Cell Proliferation

in Dental Epithelial Cells.

Front. Cell Dev. Biol. 8:156.

doi: 10.3389/fcell.2020.00156
Many genes encoding growth factors, receptors, and transcription factors are induced by the epithelial-mesenchymal interaction during tooth development. Recently, numerous functions of microRNAs (miRNAs) are reportedly involved in organogenesis and disease. miRNAs regulate gene expression by inhibiting translation and destabilizing mRNAs. However, the expression and function of miRNAs in tooth development remain poorly understood. This study aimed to analyze the expression of miRNAs produced during tooth development using a microarray system to clarify the role of miRNAs in dental development. miR-1 showed a unique expression pattern in the developing tooth. miR-1 expression in the tooth germ peaked on embryonic day 16.5, decreasing gradually on postnatal days 1 and 3 . An in situ hybridization assay revealed that miR1 is expressed at the cervical loop of the dental epithelium. The expression of miR-1 and connexin (Cx) 43, a target of miR-1, were inversely correlated both in vitro and in vivo. Knockdown of miR-1 induced the expression of Cx43 in dental epithelial cells. Interestingly, cells with miR-1 downregulation proliferated slower than the control cells. Immunocytochemistry revealed that $\mathrm{C} \times 43$ in cells with miR-1 knockdown formed both cell-cell gap junctions and hemichannels at the plasma membrane. Furthermore, the rate of ATP release was higher in cells with miR-1 knockdown than in control cells. Furthermore, Cx43 downregulation in developing molars was observed in Epiprofinknockout mice, along with the induction of miR-1 expression. These results suggest that the expression pattern of $\mathrm{Cx} 43$ is modulated by miR-1 to control cell proliferation activity during dental epithelial cell differentiation.

Keywords: dental development, differentiation, microRNA, cell proliferation, connexin 43

\section{INTRODUCTION}

Tooth development is regulated by a series of epithelial-mesenchymal tissue interactions to form morphologically optimized functional cusps. Tooth development can be divided into four stages: initiation [embryonic day (E) 11.5], bud (E13.5), cap (E15.5), and bell (E17.5) (Nakamura et al., 2004). Studies using knockout and transgenic mice have elucidated the complex genetic regulation of tooth development and growth to control the crown of teeth. However, each tooth shows 
morphologically different shape as well as size oral cavity in humans. To fabricate these varied tooth shapes, the regulatory mechanism of each gene involved in tooth development may be different.

microRNAs (miRNAs or miRs) are small non-coding RNAs (18-22 nt) involved in regulating post-transcriptional gene expression by controlling the stability and translation of mRNAs in organogenesis and diseases (Gregory et al., 2006).

Although millions of promoter activity regions have been identified in the human genome via FANTOM CAGE analysis at Riken, the mechanisms underlying transcriptional regulation in individual non-coding RNAs, small RNAs, and miRNAs remain unknown (Brennecke et al., 2005; Kawaji et al., 2011). In silico analysis predicted that conserved vertebrate miRNAs target more than 400 regulatory genes (Bartel, 2004, 2009). Diverse miRNA functions have been reported in essential cellular phenomena including cell proliferation, differentiation, and celltype specification in studies on dicer-null mice. Dicer is required for the processing of most miRNAs and for digesting long dsRNAs into small interfering RNAs (Bernstein et al., 2003; Kloosterman and Plasterk, 2006).

The dental phenotypes of epithelial-specific conditional knockout dicer mice using cytokeratin 14-Cre $\left(\mathrm{Dcr}^{\mathrm{K} 14-/-}\right)$ and Pitx2-Cre (Dcr Pitx2-/-) have been reported (Cao et al., 2010; Michon et al., 2010). Germline pathogenic variation in DICER1 causes dental abnormalities. Conditional inhibition of miRNA production in the epithelium of the tooth germ reportedly resulted in significant aberrations in molar cusp patterning and grooves on the labial surface of the incisors. Furthermore, $\mathrm{Dcr} \mathrm{K}^{\mathrm{14}-/-}$ mice displayed impaired dental epithelial cell differentiation into ameloblasts and deficient enamel formation both in molars and incisors. $D c r^{P i t x 2-/-}$ mice had relatively more severe phenotypes than $D c r^{K 14-/-}$ mice. In $D c r^{K 14-/-}$ and $D_{c r}{ }^{P i t x 2-/-}$ mice, the entire miRNA production was blocked in epithelial cells; hence, limited information is available regarding the expression and roles of miRNA in tooth development.

One of the miR-1 target genes is Gja-1 (gap junction protein, alpha-1) which encodes connexin 43 (Cx43) gap junction proteins (Yang et al., 2007; Xu et al., 2012). Cx43 is expressed on the plasma membrane of cells and forms a connexon: a protein complex comprising six connexin proteins. The connexon structure is essential for the functioning of gap junctions. Cx43 was initially identified as a tumor suppressor gene owing to an inverse correlation between tumor malignancy and Cx43 expression in tumor cells (Plante et al., 2011). Although the mechanism through which Cx43 inhibits cell proliferation remains unknown, the connexin hemi-channel potentially contributes to intracellular ATP release to the extracellular milieu (Batra et al., 2012). Depletion of intracellular ATP potentially suppresses cell growth (Cheng et al., 2014; Chi et al., 2014).

Oculodentodigital dysplasia (ODDD) is an autosomal dominant human disease caused by mutations in GJA-1, which encodes Cx43. ODDD syndrome is characterized by small eyes, abnormal face shape, syndactyly of the fourth and fifth fingers and toes, and severe hypoplastic enamel (van Es et al., 2007; Porntaveetus et al., 2017).
Epiprofin (Epfn) is a master gene in ameloblast differentiation, belonging to the Sp family of transcription factors (Nakamura et al., 2011; Aurrekoetxea et al., 2016). Epfn is expressed in developing teeth, hair follicles, skins, limb, and genitals. Epfndeficient mice have supernumerary tooth formation, enamel hypoplasia, abnormal hair follicle formation, skin abnormality, and oligodactyly with a distal bifurcation of synostotic digits and cutaneous syndactyly (Nakamura et al., 2008, 2014; Talamillo et al., 2010). The expression patterns of Epfn and Gja-1 are overlapped especially in developing teeth and limbs (Richardson et al., 2004; Nakamura et al., 2008; Talamillo et al., 2010). During limb and tooth development, Epfn-deficient mice develop similar phenotypes to those of Gja-1 null mice and are used as animal models of ODDD syndrome (Richardson et al., 2004). However, ODDD patients do not present with supernumerary teeth, which is observed in Epfn-deficient mice.

A better understanding of the role of miRNAs in tooth development would elucidate their role in prominent diseases including ODDD and further the understanding of this complex developmental process. Herein, we analyzed the expression profiles of miRNAs during tooth development, particularly focusing on miR-1. We used knockdown miR-1 cells and molecular methods to elucidate the association between miR-1 expression and $\mathrm{Cx} 43$ at various stages of tooth development.

\section{MATERIALS AND METHODS}

\section{Cell Culture and Transfection of the miR-1 Knockdown Probe}

The rat-derived dental epithelial cell line, SF2, was cultured at $37^{\circ} \mathrm{C}$ under $5 \% \mathrm{CO}_{2}$ in Ham F-12/Dulbecco's modified Eagle's medium supplemented with $10 \%$ fetal bovine serum (Nakamura et al., 2017). To knockdown miR-1 in SF2 cells, we used LNA miR-1 knockdown probes labeled with FITC or non-labeled probes (Exiqon, Denmark), with five nucleotides or deoxynucleotides at both ends of the antisense molecule locked (LNA; the ribose ring is constrained by a methylene bridge between the $2^{\prime}$-O- and the $4^{\prime}$-Catoms) (Jorgensen et al., 2010). The sequence of LNA-antimiR- 1 was $5^{\prime}$-ACTTCTTTACATTCC$3^{\prime}$. A scrambled sequence was used as a negative control: $5^{\prime}$-ATCTTACTTATCCTC-3'. miR-1 knockdown or scramble control probes $\left(5^{\prime}\right.$-ACGTCTATACGCCCA- $\left.3^{\prime}\right)$ were transfected using lipofectamine 2000 and PLUS reagent (Thermo Fisher Scientific, CA, United States).

\section{miRNA Extraction and miRNA Array Analysis}

Small RNAs were purified from ICR mice developing molars on E16.5, post-natal 1 day (P1), and P3 using the mirVana miRNA isolation $\mathrm{kit}^{\mathrm{TM}}$ system (Ambion, TX, United States) in accordance with the manufacturer's instructions. To identify the differentially expressed miRNAs in the developing tooth, we utilized the Genopal ${ }^{\mathrm{TM}}$ miRNA gene chip system. Preparation of small-sized RNA, hybridization, and signal detection were performed in accordance with the Genopal protocol (Mitsubishi 
Rayon, Japan) (Jorgensen et al., 2010). The raw data will be made available without undue reservation to any qualified researcher. The miRNA expression data for this study are available in the Gene Expression Omnibus (GEO) under accession number GSE141608.

\section{Cell Proliferation Assay}

Cell proliferation was measured using a WST assay (Dojindo, Japan) and via incorporation of 5-bromo-2'-deoxyuridine (BrdU). Four-thousand SF2 cells incubated with Anti-BrdU antibodies were visualized using an Alexa 594-conjugated secondary antibody (Thermo Fisher Scientific) (Nakamura et al., 2016). Nuclei were stained with Hoechst 33342 dye (Thermo Fisher Scientific). A BrdU incorporation assay was performed using 5-bromo-2'-deoxyuridine labeling and a detection kit (Sigma-Aldrich, MO, United States) in accordance with the manufacturer's instructions (Ibarretxe et al., 2012). SF2 cells transfected with either knockdown miR-1 or scramble probes were cultured on a glass slide for $24 \mathrm{~h}$. Before fixation with glycine/methanol, cells were labeled with BrdU for $1 \mathrm{~h}$. BrdU was detected in accordance with the manufacturer's protocol.

\section{In situ Hybridization, Immunohistochemistry, and Immunocytochemistry}

FITC-labeled single-strand locked nucleic acid (LNA) RNA probes for miR-1 and U6 were obtained from Exiqon (Qiagen, Germany). LNA probes were hybridized in accordance with the manufacturer's instructions. Frozen tissue sections were obtained from heterozygous or homozygous Epfn-deficientmouse heads (E16.5, P1, and P3) containing molars, and were placed on RNase-free glass slides (Nakamura et al., 2008). The SF2 cells on glass slides were transfected with either miR-1 knockdown or scramble probes and cultured for $48 \mathrm{~h}$ and fixed with $4 \%$ paraformaldehyde in PBS for 5 min. Primary anti-connexin 43 (1:400 dilution, Santacruz Biotechnology, CA, United States), anti-E-cadherin (1:200 dilution, BD Pharmingen, CA, United States), and antiEpiprofin antibodies (1:400 dilution) were visualized using Alexa 488 or Alexa 594-conjugated secondary antibodies (1:500 dilution, Thermo Fisher Scientific) (Nakamura et al., 2008). Nuclear staining was performed with Hoechst dye (Thermo Fisher Scientific). Immunohistochemistry, in situ hybridization, or immunocytochemistry were performed independently in triplicate. Images for immunohistochemistry, in situ hybridization, and immunocytochemistry were captured using a BZ-8000 microscope (KEYENCE, Japan). Histological analysis was performed using BZ analyzer (KEYENCE). The experimental animal protocol for maintaining mice was approved by the Institutional Animal Care Committee of Tohoku University (No. 2017DnA-045).

\section{Western Blotting}

SF2 cells transfected with either knockdown miR-1 or scramble probes were lysed in M-per buffer plus protease inhibitor cocktail (Sigma-Aldrich). The samples were centrifuged (14000 rpm,
$5 \mathrm{~min}, 4^{\circ} \mathrm{C}$ ), and the supernatants were diluted in NuPage LDS buffer and then separated on a 4-20\% NuPage Bis-tris gradient gel (Thermo Fisher Scientific). Separated proteins were electrotransferred on to PVDF membranes (Thermo Fisher Scientific). After blocking with 3\% non-fat skim milk in PBS, the membrane was probed with anti-Cx43 (Abcam, United Kingdom) or anti$\beta$-actin primary antibodies (1:100 dilution, Abcam) in PBS. The primary antibodies were detected using HRP-conjugated antirabbit IgG secondary antibodies (1:500 dilution, Thermo Fisher Scientific) using an ECL kit (Amersham Biosciences Co., NJ, United States) and a LAS $4000 \mathrm{UV}$ mini system (Fujifilm-GE healthcare, Japan).

\section{Real-Time RT-PCR Analysis}

Small RNAs were purified from homogenized mouse tissue (ICR, Epfn ${ }^{ \pm}, \mathrm{Epfn}^{-/-}$) of developing molars on E16.5, P1, and P3, using a miRVanaTM miRNA isolation kit (Thermo Fisher Scientific) and a Micro Smash ${ }^{\mathrm{TM}}$ (TOMY, Japan) in accordance with the manufacturer's instructions. miRNA cDNA was synthesized using the TaqMan ${ }^{\mathrm{TM}}$ miRNA Reverse Transcription Kit (Thermo Fisher Scientific) with mmu-miR-1. Real-time PCR was performed using a standard TaqMan ${ }^{\mathrm{TM}}$ PCR protocol on an Applied Biosystems StepOne ${ }^{\mathrm{TM}}$ real-time PCR System (Thermo Fisher Scientific). The reaction protocol was as follows: $95^{\circ} \mathrm{C}$ for $10 \mathrm{~min}$, followed by 40 cycles at $95^{\circ} \mathrm{C}$ for $15 \mathrm{~s}$ and $60^{\circ} \mathrm{C}$ for $1 \mathrm{~min}$.

Total RNA was extracted from mouse molars in Epfn \pm or $-/-$ mice using the ISOGEN II reagent (Nippon gene, Japan). After $2 \mathrm{U}$ of DNaseI (Sigma-Aldrich) treatment, $1 \mu \mathrm{g}$ of total RNA was reverse-transcribed using SuperScript ${ }^{\circledR}$ VILO $^{\text {TM }}$ Master Mix (Thermo Fisher Scientific) to generate cDNA, which was used as a template for PCR reactions with gene-specific primers (Table 1). For semiquantitative RT-PCR, cDNA was amplified with an initial denaturation step of $95^{\circ} \mathrm{C}$ for $3 \mathrm{~min}$, then $95^{\circ} \mathrm{C}$ for $30 \mathrm{~s}, 60^{\circ} \mathrm{C}$ for $30 \mathrm{~s}$, and $72^{\circ} \mathrm{C}$ for $30 \mathrm{~s}$ for 30 cycles, and a final elongation step at $72^{\circ} \mathrm{C}$ for $5 \mathrm{~min}$. The RT-PCR products were separated on a $2 \%(\mathrm{w} / \mathrm{v})$ agarose gel in Tris-acetate EDTA buffer, stained with SYBR ${ }^{\mathrm{TM}}$ safe DNA Gel Stain (Thermo Fisher Scientific), and viewed under UV light, using a LAS 4000 UV mini system (Fujifilm-GE healthcare).

\section{Quantification of ATP Release in miR-1 Knockdown SF2 Cells}

The amount of ATP released was determined via luminometry, using an ATP detection kit (Promega, WI, United States)

TABLE 1 | Primer sequence using RT-PCR analysis.

\begin{tabular}{ll}
\hline Gene & Sequence \\
\hline mEpiprofin & 5'-TCTCACTATTCACCCTCCCCTG-3' \\
mGja-1 & 5'-ACCTCATCTCTGCTTCTCTCCG-3' \\
& 5'-TTGGGGGGTGTITGGGATAGC-3' \\
mHPRT & 5'-TTAGCGGGGATGTAGGACAACCTG-3' \\
& 5'-GCGTCGTGATTAGCGATGATGA-3' \\
& 5'-GTCAAGGGCATATCCAACAACA-3'
\end{tabular}


in accordance with the manufacturer's instructions. Briefly, SF2 cells transfected with either knockdown miR-1 or scramble probes were seeded at $1 \times 10^{3}$ cells/well in a 96well plate and incubated for $24 \mathrm{~h}$. The supernatant was harvested and assayed with luciferase/luciferin. Released ATP was relatively quantified by detecting the fluorescence signal emitted in the luciferase-mediated reaction of $\mathrm{D}$ luciferin with ATP into D-oxyluciferin and measured using a GloMax ${ }^{\circledR}$ 20/20 Luminometer (Turner BioSystems, CA, United States).

\section{Statistical Analysis}

Each experiment was performed independently in triplicate. Data are presented as mean \pm standard deviation (SD) values. The Student's $t$-test and the one-way analysis of variance (ANOVA) were used. When the standard deviations were significantly different between groups, the Kruskal-Wallis non-parametric ANOVA test were used. A $P$-value of $<0.05$ was considered significant. Data analysis was performed using Prism $8^{\mathrm{TM}}$ software (GraphPad Software, CA, United States).

\section{RESULTS}

\section{miRNA Expression Profiling During Tooth Development}

Because the shape of the crown is determined after the cap stage, and dynamic cytodifferentiation occurs after the bell stage, we selected the characteristic stages of molar development at E16.5, P1, and P3. We prepared total RNA from tooth germ at E16 and newborn stages plus postnatal (P) 3 days, representing mature tooth development, for microarray-based comparison. The miRNA microarray data of these three stages of tooth development were compared (Supplementary Figure S1).

Fifty-seven miRNAs were significantly upregulated or downregulated during tooth development. Interestingly, miR1, miR-376a, miR-124a, and miR-127 displayed particularly dynamic expression changes during tooth development (Figure 1A). miR-1 was strongly expressed at E16 and P1 but drastically reduced at P3 (Figure 1A). As expected, the expression profiles of miR-133a and miR-206, which are cluster miRNAs with miR-1, were synchronized with that of miR-1 (Figure 1A).

\section{TaqMan ${ }^{\mathrm{TM}}$ Analysis of miR-1 Expression During Tooth Development}

In the heatmap generated on the basis of the miRNA microarray during tooth development, miR-1 was upregulated on E16.5 and was significantly downregulated on P3. Therefore, we validated miR-1 expression in the tooth germ at these stages using the TaqMan $^{\text {TM }}$ system. As expected, miR-1 expression was detected using TaqMan ${ }^{\mathrm{TM}}$ primer and probe system in E16 tooth germ cDNA (Figure 1B). We observed a 90\% reduction in miR-1 expression in the P3 tooth germ compared to in the E16.5 tooth germ (Figure 1B). These results validate the present microarray results and confirm the unusual expression pattern of miR-1 during tooth development.
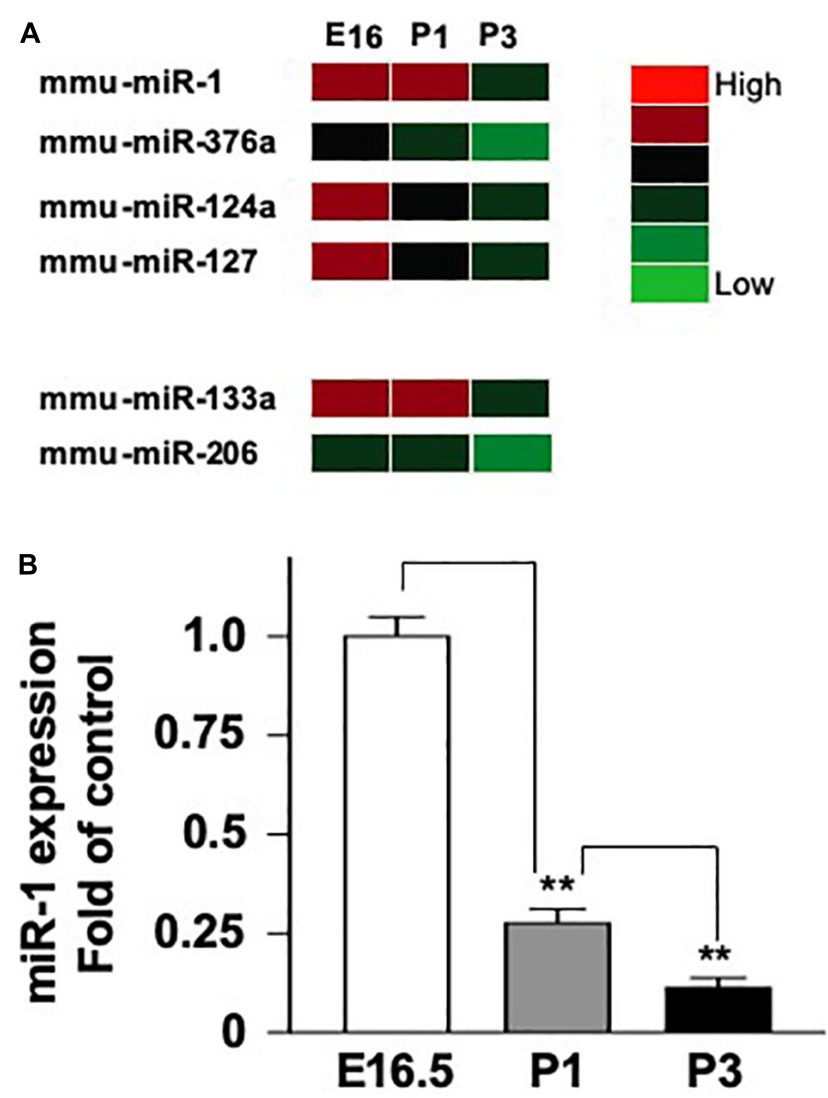

C

\section{E16.5 Molar}

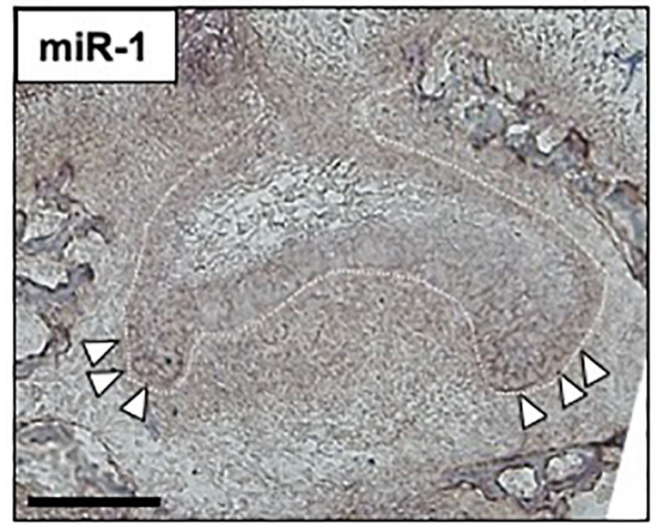

FIGURE 1 | The expression of miR-1 during tooth development. (A) miRNA microarray data at three stages (E16.5, P1, P3) during tooth development. High expression is indicated by red-colored bars, and low expression is indicated by light green colored bars. Heat map analysis of miRNA-1, -376a, $-124 a$, and -127 , which were down-regulated with tooth developmental stages. (B) Real-time PCR analysis of miR-1 expression at E16.5 and P3. miR-1 was highly expressed at E16.5 in the tooth germ but had significantly lower expression at P3 than E16.5. Data are presented as mean \pm standard deviation (SD) values. The statistical analysis was done by the Kruskal-Wallis non-parametric ANOVA test ( $\left.{ }^{\star \star} P<0.01\right)$. (C) miR-1 was detected at both sides of the cervical loop in the E16.5 tooth bud (white arrowheads). The white dot line indicates the margin of the dental epithelium and the mesenchyme. $n=3$, Scale bar $100 \mathrm{~mm}$. 


\section{The Tissue-Specific Expression Pattern of miR-1 During Tooth Development}

We confirmed that the miR-1 expression was significantly altered during tooth development in homogenized tissue; hence, we performed tissue-specific in situ hybridization of miR1 to identify the spatial pattern of expression during tooth development. miR-1 in the developing molar on E16.5 was primarily localized in the cervical loop, which contains highly proliferating dental epithelial cells (Figure 1C), suggesting that miR-1 potentially regulates cell proliferation in dental epithelial cells.

\section{Negative Regulation of Cx43 Expression by miR-1 in Dental Epithelial Cells}

One of the known targets of miR-1 is Gja-1, which encodes the Cx43 gap junction protein (Figure 2A). miR-1 interferes with the translation of Gja-1 into $\mathrm{Cx} 43$ in numerous cell types. Hence, we investigated the effect of miR-1 on $\mathrm{Cx} 43$ production, using a dental epithelial cell line, SF2, using a miR-1 knockdown system. The efficiency of incorporation of small interfering miR-1 (simiR-1) and control (scramble) siRNA was approximately $70 \%$ of the total cells (Supplementary Figure S2). Cx43 expression in the control (Scramble in Figure 2B) was detected as a single band at the expected size. The miR-1 knockdown cells, either by si-miR1 or si-miR1-FITC conjugated probe, expressed $\mathrm{Cx} 43$ at a higher level than that of the control (Figure 2B). Band intensities (Figure 2B) were quantified and normalized to the expression levels of the internal control $\beta$-actin. The expression level of $\mathrm{Cx} 43$ in miR-1 knockdown cells, by either si-miR1 or si-miR1-FITC conjugated probe, increased by twofold compared to that of the control (scramble transfected cells) (Figure 2C).

\section{The Role of miR-1 in Dental Epithelial Proliferation}

To investigate the cellular functions of miR-1, we measured cell proliferation after blocking miR-1 expression with simiR1. We observed a reduction in cell proliferation activity via the WST- 8 assay in miR-1 knockdown cells compared to non-treated siRNA or scramble control oligo-transfected cells (Figure 3A). miR-1 thus positively regulates proliferation in dental epithelial cells. To confirm these results, we also assessed cell proliferation using a BrdU incorporation assay. si-miR1 labeled with FITC, visualized as a green signal, incorporated less BrdU (red) than the control (Figure 3B, arrows). To quantify these results, we enumerated the BrdU/FITC double-positive cells and probe-incorporating FITC-positive cells. Approximately $35 \%$ of scramble FITC positive cells were positive for BrdU (red) (Figure 3B, arrowheads). However, approximately $25 \%$ of cells with miR-1 knockdown-FITC probes also incorporated BrdU (red), indicating a $10 \%$ reduction in the number of BrdU/FITCdouble-positive cells upon miR-1 knockdown (Figure 3B). Furthermore, no significant differences were observed in nuclear morphology between scramble and si-miR-1-transfected cells, suggesting that miR-1 knockdown diminished cell proliferation without inducing cell death (Figure 3B). Based on these results,

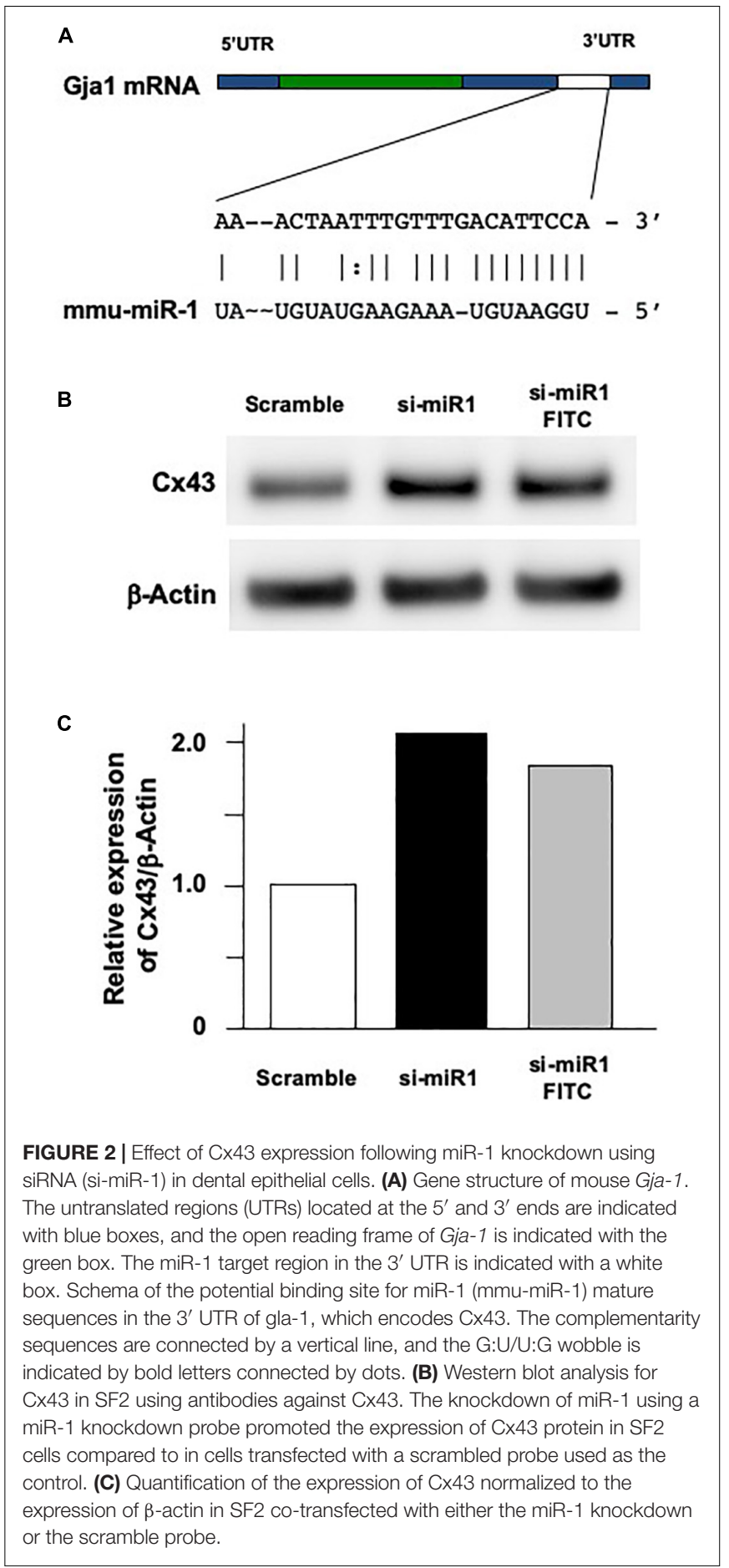

the expression of miR-1 appears to be required for proper cell proliferation during tooth development.

\section{The Regulation of Cx43 Cellular Localization by miR-1 in Dental Epithelial Cells}

Cx43 cellular localization was analyzed in SF2 cells after miR1 knockdown. While in scramble (control) cells, $\mathrm{Cx} 43$ was 


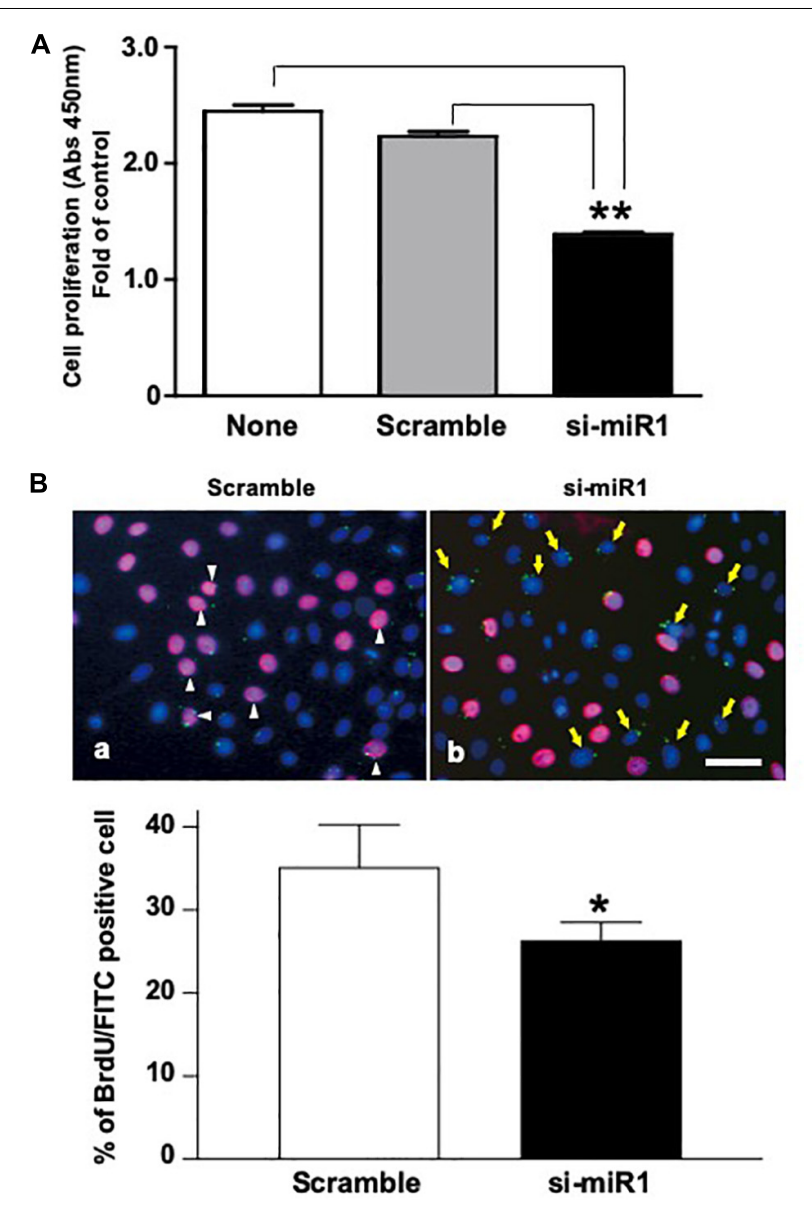

FIGURE 3 | Promotion of cell proliferation in miR-1-knockdown SF2 cells using si-miR1 probes. (A) Cell proliferation assay. SF cells transfected with miR-1 knockdown had reduced cell proliferation compared to cells transfected with scramble, or no probe (denoted 'none'). Data are presented as mean \pm standard deviation (SD) values. The statistical analysis was done by the Kruskal-Wallis non-parametric ANOVA test $\left.{ }^{\star \star} P<0.01\right)$ (B) BrdU incorporation assay with FITC labeled miR-1 or scramble probes. The cell nuclei that had incorporated BrdU were stained red, while miR-1 knockdown (si-miR1) (yellow arrows in b) or scramble (white arrowheads in a) probe-transfected cells were stained green with FITC. Nuclear staining was performed with Hoechst 33342 (blue). Scale bar 100 mm There was a 10\% reduction in the amount of BrdU incorporation in SF cells that were co-transfected with si-miR1-FITC compared to that of scramble-FITC. Data are presented as mean \pm standard deviation (SD) values. The statistical analysis was done by the Student's $t$-test $\left({ }^{\star} P<0.05\right)$.

expressed on the plasma membrane at the cell-cell junction, indicating that $\mathrm{Cx} 43$ forms part of the gap junction. In miR1 knockdown SF2 cells, Cx43 was localized on the plasma membrane at the sides that were not adjacent to other cells and at the cell-cell junction (Figure 4A), suggesting that excess $\mathrm{Cx} 43$ induced by knockdown of miR-1 also accumulates at the hemichannels. Cultured astrocytes are known to release glutamate and ATP in divalent cation-free media via gap junction hemichannels (Stout et al., 2002). Hence, we quantified ATP release from dental epithelial cells transfected with si-miR1 or scramble control probes. The amount of

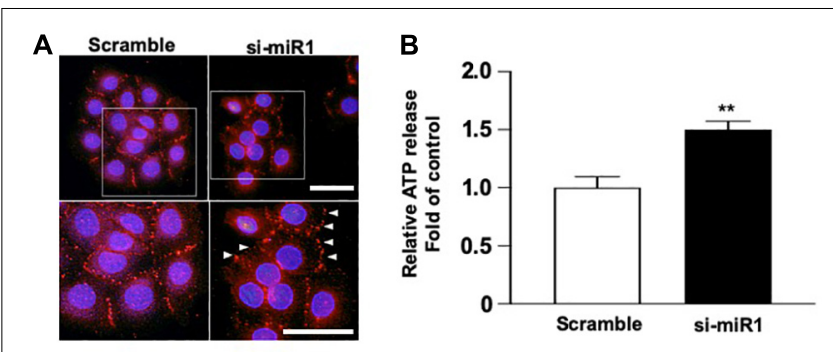

C

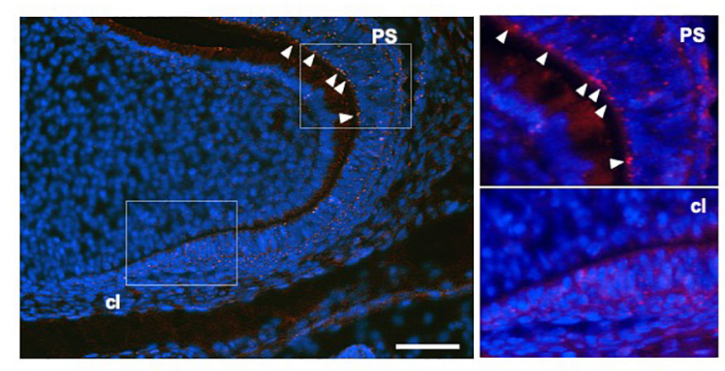

D
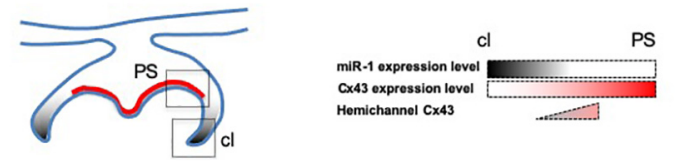

FIGURE 4 | Dynamic changes in the cellular localization of Cx43 following miR-1 knockdown. (A) Immunocytochemistry analysis of $\mathrm{Cx} 43$ in control (Scramble) and miR-1 knockdown (si-miR1) dental epithelial cells. Higher magnification images are shown in the boxed area below. Arrowheads indicate hemichannel formation by $\mathrm{C} \times 43$ following the knockdown of miR-1. Scale bar $50 \mathrm{~mm}$. (B) Effect of miR-1 on the release of extracellular ATP in control dental epithelial cells (Scramble) and miR-1 knockdown cells (si-miR1). Data are presented as mean \pm standard deviation (SD) values. The statistical analysis was done by the Student's $t$-test $\left({ }^{\star \star} P<0.01\right)$. (C) The expression pattern of hemichannel $\mathrm{C} \times 43$ in developing molars (P1). Differentiating presecretory ameloblasts express hemichannel Cx43 (arrowheads). Gap junctions were formed in dental epithelial cells at the cervical region. Scale bar $100 \mathrm{~mm}$. (D) Schematic representation of miR-1 and Cx43 localization in the developing molar. An inverse gradient of expression was observed between miR-1 and $\mathrm{C} \times 43$, as well as in the distribution of hemichannel $\mathrm{C} \times 43$ in the developing molar. PS, presecretory ameloblast; cl, cervical loop.

extracellular release of ATP from miR-1 knockdown dental epithelial cells was significantly higher than that in control cells transfected with the scramble probe (Figure 4B). miR1 may therefore regulate cell proliferation by regulating $\mathrm{Cx} 43$ production and $\mathrm{Cx} 43$ hemichannel formation. To verify our in vitro analysis, we observed hemichannel $\mathrm{Cx} 43$ localization in developing molars. Cx43 was expressed in both dental epithelial cells and presecretory ameloblasts, which are postmitotic cells. Cx43 localized on the cellular plasma membrane adjacent to other cells, indicating that $\mathrm{Cx} 43$ forms gap junctions (Figure 4C). Interestingly, Cx43 was downregulated in dental epithelial cells at the cervical region, where miR1 is expressed, compared with the inner dental epithelium (Figure 1C), suggesting that miR-1 localized expression may inhibit Cx43 expression. Cx43 was also detected at the sides of the basal membrane in addition to the cell-cell contact 


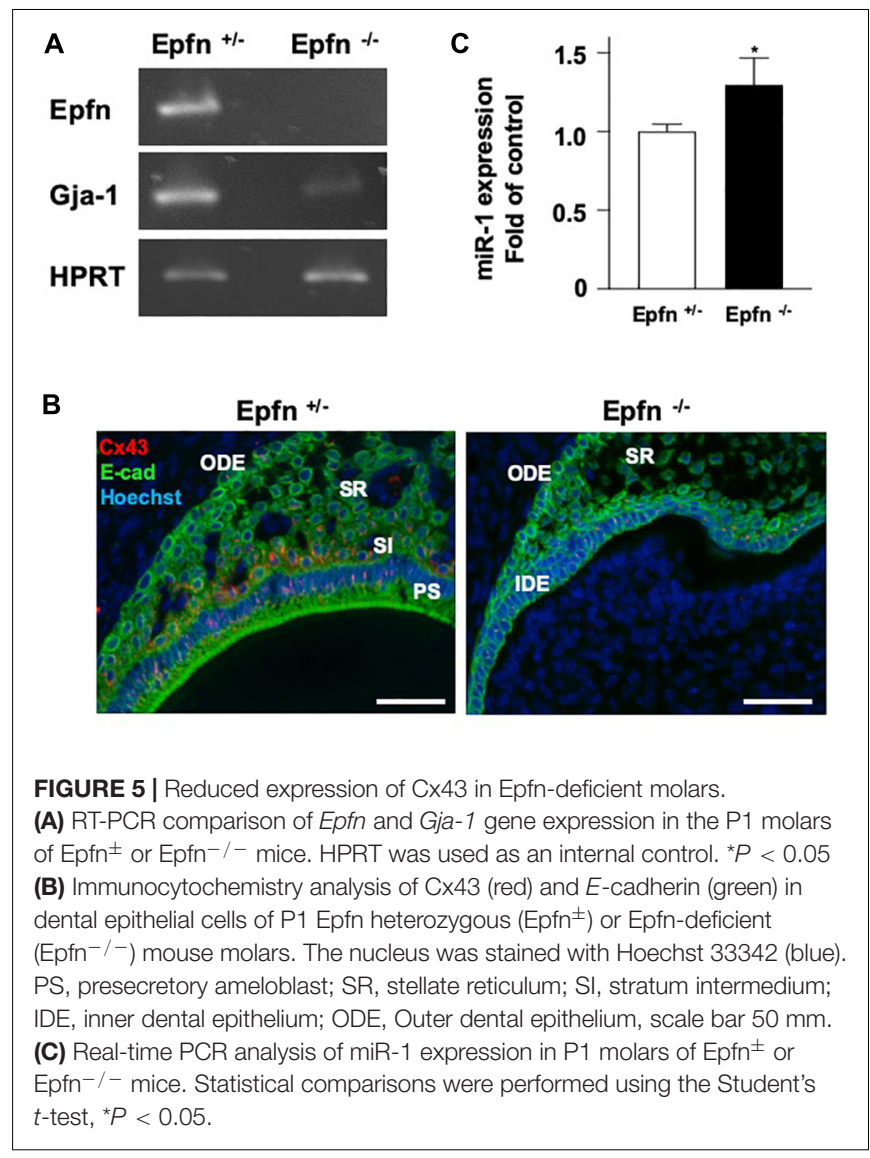

plasma membrane (Figure 4C, arrowheads). Cx43 localized at the sides of the basal membrane in presecretory ameloblasts did not form gap junctions, which are expressed by other cells (Figure 4C). These results suggest that $\mathrm{Cx} 43$ expressed by presecretory ameloblasts forms both gap junctions and hemichannels, and the formation of hemichannels may inhibit cell proliferation during ameloblast differentiation, as shown in Figure 4D.

\section{The Induction of miR-1 Expression in Molars of Epfn-Deficient Mice}

The expression of Gja-1, encoding Cx43, was greatly reduced in Epfn ${ }^{-/}$molars (Figure 5A). Immunohistochemical analysis with an anti-Cx43 antibody revealed reduced expression of Cx43 in Epfn ${ }^{-/-}$mouse molars compared to in $\mathrm{Epfn}^{ \pm}$mice (Figure 5B). Because Epfn is a master gene in ameloblast differentiation, dental epithelial cell differentiation is completely blocked Epfn ${ }^{-/}$mouse molars. Next, we measured the expression of miR-1 in undifferentiated dental epithelial cells in $\mathrm{Epfn}^{-/-}$mouse molars using TaqMan ${ }^{\mathrm{TM}}$ and observed increased miR-1 expression in Epfn ${ }^{-/}$molars compared to Epfn ${ }^{ \pm}$molars (Figure 5C). These results support the inverse correlation of the expression between miR-1 and Cx43 observed in developing molars, and suggest that the attenuation of miR-1 is important for the differentiation of dental epithelial cells into ameloblasts.

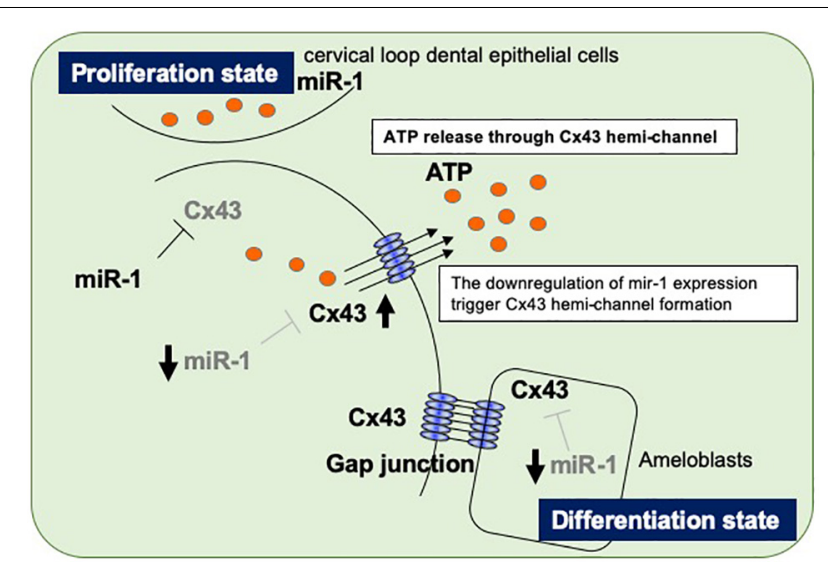

FIGURE 6 | Proposed model of the regulatory mechanism in ameloblasts by miR-1-mediated Cx43 expression during dental epithelial cell differentiation. Dental epithelial cells at the cervical loop area proliferate rapidly and express miR-1, which block Cx43 expression. Proliferating dental epithelial cells start to express $\mathrm{Cx} 43$ by the attenuation of miR-1 expression and form hemichannel $\mathrm{C} \times 43$ to release intracellular ATP. The inverse expression between miR-1 and Cx43 is observed during dental epithelial cell differentiation into ameloblasts.

\section{DISCUSSION}

The regulation of organogenesis by miRNAs has been studied using dicer-conditional $\mathrm{KO}$ mice cross-mated with specific interest Cre transgenic mice. Cytokeratin 14-Cre and Pitx2Cre mice have been used to generate dicer knockdown mice in epithelial cell lineages, while Wnt1-Cre mice have been used to generate dicer knockdown mice in neural crest-derived mesenchyme lines (Cao et al., 2010; Michon et al., 2010). $D c r^{K 14-/-}$ mice display impaired ameloblast differentiation and enamel formation with abnormal crown shapes (Michon et al., 2010), and the dental epithelium in $\mathrm{Dcr}^{\text {Pitx2 }-/-}$ mice forms extra cell niches, which develop extra incisors (Cao et al., 2010). DcrWnt1/- mice, which have defective miRNA production in neural crest derived-cell lineages, have extremely severe craniofacial abnormalities and missing tooth buds (Cao et al., 2010). These reports imply that miRNAs play essential roles in normal tooth development. To date, there have been reports of many different miRNAs expressed during tooth development, both in the dental epithelium and in the mesenchyme, but the function of specific miRNAs remains unclear. In the present study, we performed high-throughput analysis of miRNAs expressed during tooth development and focused on characterizing the role of miR-1 (Figure 6). Connexin 43 (Cx43), a gap junction protein, is a target molecule of miR1 , and no connexins other than $\mathrm{Cx} 43$ have been identified as a target gene of miR-1 (Klotz, 2012). Our results suggest that Gja-1 is a target gene of miR-1 in dental epithelial cells, as in cardiomyocytes (Yang et al., 2007). Although connexins including $\mathrm{Cx} 26, \mathrm{Cx} 32$, and $\mathrm{Cx} 43$ are expressed in dental epithelial cells, Cx43 knockdown in developing teeth causes a severe enamel hypoplasia, suggesting that Cx26 and Cx32 cannot functionally compensate for Cx43 (Toth et al., 2010). 
Cx43 is most predominantly expressed during development especially in dental epithelial cells; however, it is downregulated in mature adult teeth but expressed in carious teeth localized at odontoblast processes owing to the obliteration of ameloblasts after the enamel formation in developing teeth (About et al., 2002; Ibuki et al., 2002). Gap junctions are made of two hemichannels from two neighboring cells across the extracellular gap. Each hemichannel, or connexon, is constructed from six connexin proteins. Standalone hemichannels can also be present in cells. These hemichannels have a low opening probability that is increased under various physiological and pathological conditions (Saez et al., 2005; Spray et al., 2006). We hypothesize that, besides mediating cell-cell communication, undocked connexin channels allow communication between the intracellular and extracellular milieu, thereby playing an important role in paracrine communication during tooth development. Recently we reported that pannexin 3 (Panx3), a hemi-channel protein, is also preferentially expressed in the tooth germ, controls cell proliferation and differentiation in dental mesenchymal cells, which differentiate into dentin-forming odontoblasts (Iwamoto et al., 2017). Panx3 hemichannels are locally expressed in pre-odontoblasts. Panx3 blocks cell proliferation and promotes differentiation into odontoblasts in pre-odontoblasts by releasing intracellular ATP to the extracellular space. The tooth is covered with epithelial-derived enamel and mesenchyme-derived dentin to confer physical hardness. These two hard tissues are distinctly built up in daily cycles by the layer structures to accumulate enamelor dentin-matrices (Kawasaki et al., 1979). The regulation of dental cell differentiation should be controlled simultaneously to create the enamel or dentin surface. Although there are differences between dental epithelial cells and mesenchymal cells, there may be a shared switching mechanism from the cell proliferative state to the differentiation state in the developing tooth. Cell-cell communication proteins, such as Cx43 and Panx3, may play roles in this mechanism in ameloblasts and odontoblasts, respectively. It may be effective to control in ameloblasts, not by transcription, but by post-transcriptional miRNAs with ATP release to stop the proliferation of ameloblasts and switch to differentiation of pre-ameloblasts on the tooth surface. In fact, ameloblast cell division does not completely occur during the enamel layer structure formation; it is formed one layer a day from the inside to the outside of the tooth. The present results report an increase in the amount of ATP release from dental epithelial cells with an miR-1 knockdown. Five groups of channels have been identified as ATP release channels, such as connexin hemichannels, pannexin 1, calcium homeostasis modulator 1 (CALHM1), volume-regulated anion channels (VRACs), and maxi-anion channels (MACs) (Liu et al., 2008; Kinnamon and Finger, 2013; Mikolajewicz et al., 2018). Furthermore, the classical exocytosis and non-vesicular mechanisms of cellular ATP release have been reported in various cell types. Studies on the route of ATP release from miR-1 knockdown-dental epithelial cells are currently underway. The present results show that the increase in ATP release in miR-1 knockdown-dental epithelial cells was 18 $\alpha$-GA, a global connexin inhibitor (data not shown) (Yamada et al., 2016).
Thus, an increase in extracellular ATP release in miR-1 knockdown-dental epithelial cells may be required to activate Cx43 because Gja-1(Cx43) is the only target gene of miR-1 in the genes encoding connexin proteins. However, the role of miR-1 in the regulation of gap junctions or hemichannel $\mathrm{Cx} 43$ activity, Cx43 cellular localization, or calcium oscillations in differentiating dental epithelial cells remains unknown. Further studies are required to elucidate the regulation of dental epithelial cell proliferation and differentiation by miR-1. We previously identified epiprofin as a novel member of the $\mathrm{Sp}$ transcription factor family expressed in certain ectodermal organs including the teeth, hair follicles, nails, skin, and limbs (Nakamura et al., 2004, 2011; Talamillo et al., 2010). The phenotype of Epfn-deficient $\left(\mathrm{Epfn}^{-/-}\right)$mice is partially shared with that of ODDD in humans, resulting from Gja-1 mutations (Richardson et al., 2004; Huang et al., 2013). Our outcomes may contribute to understanding the pathogenesis of ODDD syndrome and also lead to developing novel treatments for enamel hypoplasia.

\section{CONCLUSION}

We observed an inverse expression pattern between miR1 and $\mathrm{Cx} 43$ in developing molars and went on to clarify their function in tooth development. During dental epithelial differentiation, the down-regulation of miR-1 induces $\mathrm{Cx} 43$ hemichannel formations to release intracellular ATP to the extracellular milieu, halting cell proliferation. In an enamel hypoplasia model, such as Epfn ${ }^{-/}$mice, the dysregulation of miR-1 in developing molars results in a reduction in the amount of $\mathrm{Cx} 43$. Although the transcriptional regulation of miR-1 in the developing tooth should be investigated, miR-1 plays important roles in enamel formation.

\section{DATA AVAILABILITY STATEMENT}

The datasets generated for this study can be found in the GEO https://www.ncbi.nlm.nih.gov/geo/query/acc.cgi?acc= GSE141608.

\section{ETHICS STATEMENT}

The animal study was reviewed and approved by Institutional Animal Care Committee of Tohoku University.

\section{AUTHOR CONTRIBUTIONS}

TaN and SF designed the study and provided funding for the study. ToN, TaN, and TI participated in the entire experiment, and writing and modifying the manuscript. $\mathrm{HN}$ and YY helped to solve problems throughout the experiment, and participated in the editing and revision of the manuscript. TI and $\mathrm{HN}$ participated in the ATP 
release assay experiments and statistical analysis of the data. TaN, $\mathrm{AY}, \mathrm{KS}$, and YS confirmed data to prepare the revised manuscript.

\section{FUNDING}

This study was supported by grants-in-aid for Scientific Research (KAKENHI) from the Japan Society for the Promotion of Science (No. 18K19634 to TaN).

\section{ACKNOWLEDGMENTS}

We thank Dr. Minoru Wakamori for technical advice and various suggestions. We also thank the Institute

\section{REFERENCES}

About, I., Proust, J. P., Raffo, S., Mitsiadis, T. A., and Franquin, J. C. (2002). In vivo and in vitro expression of connexin 43 in human teeth. Connect Tissue Res. 43, 232-237. doi: 10.1080/713713515

Aurrekoetxea, M., Irastorza, I., Garcia-Gallastegui, P., Jimenez-Rojo, L., Nakamura, T., Yamada, Y., et al. (2016). Wnt/beta-catenin regulates the activity of epiprofin/Sp6, SHH, FGF, and BMP to coordinate the stages of odontogenesis. Front. Cell Dev. Biol. 4:25. doi: 10.3389/fcell.2016.00025

Bartel, D. P. (2004). MicroRNAs: genomics, biogenesis, mechanism, and function. Cell 116, 281-297.

Bartel, D. P. (2009). MicroRNAs: target recognition and regulatory functions. Cell 136, 215-233. doi: 10.1016/j.cell.2009.01.002

Batra, N., Kar, R., and Jiang, J. X. (2012). Gap junctions and hemichannels in signal transmission, function and development of bone. Biochim. Biophys. Acta. 1818, 1909-1918. doi: 10.1016/j.bbamem.2011.09.018

Bernstein, E., Kim, S. Y., Carmell, M. A., Murchison, E. P., Alcorn, H., Li, M. Z., et al. (2003). Dicer is essential for mouse development. Nat. Genet. 35, 215-217. doi: $10.1038 / \mathrm{ng} 1253$

Brennecke, J., Stark, A., Russell, R. B., and Cohen, S. M. (2005). Principles of microRNA-target recognition. PLoS Biol. 3:e85. doi: 10.1371/journal.pbio. 0030085

Cao, H., Wang, J., Li, X., Florez, S., Huang, Z., Venugopalan, S. R., et al. (2010). MicroRNAs play a critical role in tooth development. J. Dent. Res. 89, 779-784. doi: 10.1177/0022034510369304

Cheng, G., Zielonka, J., McAllister, D., Tsai, S., Dwinell, M. B., and Kalyanaraman, B. (2014). Profiling and targeting of cellular bioenergetics: inhibition of pancreatic cancer cell proliferation. Br. J. Cancer. 111, 85-93. doi: 10.1038/bjc. 2014.272

Chi, Y., Gao, K., Li, K., Nakajima, S., Kira, S., Takeda, M., et al. (2014). Purinergic control of AMPK activation by ATP released through connexin 43 hemichannels - pivotal roles in hemichannel-mediated cell injury. J. Cell Sci. 127, 1487-1499. doi: 10.1242/jcs. 139089

Gregory, R. I., Chendrimada, T. P., and Shiekhattar, R. (2006). MicroRNA biogenesis: isolation and characterization of the microprocessor complex. Methods Mol. Biol. 342, 33-47.

Huang, T., Shao, Q., MacDonald, A., Xin, L., Lorentz, R., Bai, D., et al. (2013). Autosomal recessive GJA1 $(\mathrm{Cx} 43)$ gene mutations cause oculodentodigital dysplasia by distinct mechanisms. J. Cell Sci. 126, 2857-2866. doi: 10.1242/jcs. 123315

Ibarretxe, G., Aurrekoetxea, M., Crende, O., Badiola, I., Jimenez-Rojo, L., Nakamura, T., et al. (2012). Epiprofin/Sp6 regulates Wnt-BMP signaling and the establishment of cellular junctions during the bell stage of tooth development. Cell Tissue Res. 350, 95-107. doi: 10.1007/s00441-012-1459-8

Ibuki, N., Yamaoka, Y., Sawa, Y., Kawasaki, T., and Yoshida, S. (2002). Different expressions of connexin 43 and 32 in the fibroblasts of human dental pulp. Tissue Cell 34, 170-176. doi: 10.1016/s0040-8166(02)00028-9 for animal experimentation in Tohoku University Graduate school of Medicine for maintain Epiprofin targeted mice.

\section{SUPPLEMENTARY MATERIAL}

The Supplementary Material for this article can be found online at: https://www.frontiersin.org/articles/10.3389/fcell.2020.00156/ full\#supplementary-material

FIGURE S1 | Results of Genopal ${ }^{\mathrm{TM}}$ miRNA gene chip array in developing tooth germ.

FIGURE S2 | Phase and fluorescence images after transfection of FITC-labeled scramble or miR-1 knockdown probes into SF2 cells.

Iwamoto, T., Nakamura, T., Ishikawa, M., Yoshizaki, K., Sugimoto, A., IdaYonemochi, H., et al. (2017). Pannexin 3 regulates proliferation and differentiation of odontoblasts via its hemichannel activities. PLoS One 12:e0177557. doi: 10.1371/journal.pone.0177557

Jorgensen, S., Baker, A., Moller, S., and Nielsen, B. S. (2010). Robust one-day in situ hybridization protocol for detection of microRNAs in paraffin samples using LNA probes. Methods 52, 375-381. doi: 10.1016/j.ymeth.2010.07.002

Kawaji, H., Severin, J., Lizio, M., Forrest, A. R., van Nimwegen, E., Rehli, M., et al. (2011). Update of the FANTOM web resource: from mammalian transcriptional landscape to its dynamic regulation. Nucleic Acids Res. 39, D856-D860. doi: $10.1093 /$ nar/gkq1112

Kawasaki, K., Tanaka, S., and Ishikawa, T. (1979). On the daily incremental lines in human dentine. Arch. Oral. Biol. 24, 939-943. doi: 10.1016/0003-9969(79) 90221-8

Kinnamon, S. C., and Finger, T. E. (2013). A taste for ATP: neurotransmission in taste buds. Front. Cell Neurosci. 7:264. doi: 10.3389/fncel.2013.00264

Kloosterman, W. P., and Plasterk, R. H. (2006). The diverse functions of microRNAs in animal development and disease. Dev. Cell 11, 441-450. doi: 10.1016/j.devcel.2006.09.009

Klotz, L. O. (2012). Posttranscriptional regulation of connexin-43 expression. Arch. Biochem. Biophys. 524, 23-29. doi: 10.1016/j.abb.2012.03.012

Liu, H. T., Toychiev, A. H., Takahashi, N., Sabirov, R. Z., and Okada, Y. (2008). Maxi-anion channel as a candidate pathway for osmosensitive ATP release from mouse astrocytes in primary culture. Cell Res. 18, 558-565. doi: 10.1038/cr.2008. 49

Michon, F., Tummers, M., Kyyronen, M., Frilander, M. J., and Thesleff, I. (2010). Tooth morphogenesis and ameloblast differentiation are regulated by microRNAs. Dev. Biol. 340, 355-368. doi: 10.1016/j.ydbio.2010.01.019

Mikolajewicz, N., Mohammed, A., Morris, M., and Komarova, S. V. (2018). Mechanically stimulated ATP release from mammalian cells: systematic review and meta-analysis. J. Cell Sci. 21:131.

Nakamura, T., Chiba, Y., Naruse, M., Saito, K., Harada, H., and Fukumoto, S. (2016). Globoside accelerates the differentiation of dental epithelial cells into ameloblasts. Int. J. Oral. Sci. 8, 205-212. doi: 10.1038/ijos.2016.35

Nakamura, T., de Vega, S., Fukumoto, S., Jimenez, L., Unda, F., and Yamada, Y. (2008). Transcription factor epiprofin is essential for tooth morphogenesis by regulating epithelial cell fate and tooth number. J. Biol. Chem. 283, 4825-4833. doi: 10.1074/jbc.m708388200

Nakamura, T., Fukumoto, S., and Yamada, Y. (2011). Diverse function of epiprofin in tooth development. J. Oral Biosci.. 53, 22-30. doi: 10.1016/s1349-0079(11) 80032-0

Nakamura, T., Jimenez-Rojo, L., Koyama, E., Pacifici, M., de Vega, S., Iwamoto, M., et al. (2017). Epiprofin regulates enamel formation and tooth morphogenesis by controlling epithelial-mesenchymal interactions during tooth development. J. Bone Miner. Res. 32, 601-610. doi: 10.1002/jbmr.3024

Nakamura, T., Unda, F., de-Vega, S., Vilaxa, A., Fukumoto, S., Yamada, K. M., et al. (2004). The Kruppel-like factor epiprofin is expressed by epithelium of 
developing teeth, hair follicles, and limb buds and promotes cell proliferation. J. Biol. Chem. 279, 626-634. doi: 10.1074/jbc.m307502200

Nakamura, T., Yoshitomi, Y., Sakai, K., Patel, V., Fukumoto, S., and Yamada, Y. (2014). Epiprofin orchestrates epidermal keratinocyte proliferation and differentiation. J. Cell Sci. 127, 5261-5272. doi: 10.1242/jcs.15 6778

Plante, I., Stewart, M. K., Barr, K., Allan, A. L., and Laird, D. W. (2011). Cx43 suppresses mammary tumor metastasis to the lung in a $\mathrm{Cx} 43$ mutant mouse model of human disease. Oncogene 30, 1681-1692. doi: 10.1038/onc.2010. 551

Porntaveetus, T., Srichomthong, C., Ohazama, A., Suphapeetiporn, K., and Shotelersuk, V. (2017). A novel GJA1 mutation in oculodentodigital dysplasia with extensive loss of enamel. Oral. Dis. 23, 795-800. doi: 10.1111/odi. 12663

Richardson, R., Donnai, D., Meire, F., and Dixon, M. J. (2004). Expression of Gja1 correlates with the phenotype observed in oculodentodigital syndrome/type III syndactyly. J. Med. Genet. 41, 60-67. doi: 10.1136/jmg.2003.01 2005

Saez, J. C., Retamal, M. A., Basilio, D., Bukauskas, F. F., and Bennett, M. V. (2005). Connexin-based gap junction hemichannels: gating mechanisms. Biochim. Biophys. Acta 1711, 215-224. doi: 10.1016/j.bbamem.2005.01.014

Spray, D. C., Ye, Z. C., and Ransom, B. R. (2006). Functional connexin "hemichannels": a critical appraisal. Glia 54, 758-773. doi: 10.1002/glia.20429

Stout, C. E., Costantin, J. L., Naus, C. C., and Charles, A. C. (2002). Intercellular calcium signaling in astrocytes via ATP release through connexin hemichannels. J. Biol. Chem. 277, 10482-10488. doi: 10.1074/jbc.m109902200

Talamillo, A., Delgado, I., Nakamura, T., de-Vega, S., Yoshitomi, Y., Unda, F., et al. (2010). Role of Epiprofin, a zinc-finger transcription factor, in limb development. Dev. Biol. 337, 363-374. doi: 10.1016/j.ydbio.2009.11.007
Toth, K., Shao, Q., Lorentz, R., and Laird, D. W. (2010). Decreased levels of $\mathrm{Cx} 43$ gap junctions result in ameloblast dysregulation and enamel hypoplasia in Gja1Jrt/+ mice. J. Cell Physiol. 223, 601-609. doi: 10.1002/jcp.22046

van Es, R. J., Wittebol-Post, D., and Beemer, F. A. (2007). Oculodentodigital dysplasia with mandibular retrognathism and absence of syndactyly: a case report with a novel mutation in the connexin 43 gene. Int. J. Oral. Maxillofac. Surg. 36, 858-860. doi: 10.1016/j.ijom.2007.03.004

Xu, H. F., Ding, Y. J., Shen, Y. W., Xue, A. M., Xu, H. M., Luo, C. L., et al. (2012). MicroRNA- 1 represses Cx43 expression in viral myocarditis. Mol. Cell Biochem. 362, 141-148. doi: 10.1007/s11010-011-1136-3

Yamada, A., Futagi, M., Fukumoto, E., Saito, K., Yoshizaki, K., Ishikawa, M., et al. (2016). Connexin 43 is necessary for salivary gland branching morphogenesis and FGF10-induced ERK1/2 phosphorylation. J. Biol. Chem. 291, 904-912. doi: 10.1074/jbc.M115.674663

Yang, B., Lin, H., Xiao, J., Lu, Y., Luo, X., Li, B., et al. (2007). The muscle-specific microRNA miR-1 regulates cardiac arrhythmogenic potential by targeting GJA1 and KCNJ2. Nat. Med. 13, 486-491. doi: 10.1038/nm1569

Conflict of Interest: The authors declare that the research was conducted in the absence of any commercial or financial relationships that could be construed as a potential conflict of interest.

Copyright (c) 2020 Nakamura, Iwamoto, Nakamura, Shindo, Saito, Yamada, Yamada, Fukumoto and Nakamura. This is an open-access article distributed under the terms of the Creative Commons Attribution License (CC BY). The use, distribution or reproduction in other forums is permitted, provided the original author(s) and the copyright owner(s) are credited and that the original publication in this journal is cited, in accordance with accepted academic practice. No use, distribution or reproduction is permitted which does not comply with these terms. 